\title{
Sex Differences in Complaints and Diagnoses ${ }^{1}$
}

\author{
Lois M. Verbrugge ${ }^{2}$ \\ Accepted for publication: March 3, 1980
}

This paper examines male-female differences in complaints and diagnoses for ambulatory care visits. Data are from the 1975 National Ambulatory Medical Care Survey, a national probability survey of visits to office-based physicians. The results suggest that: (l) Men are often unaware of serious health problems, they delay seeking diagnosis and care for symptoms, and they hesitate to admit sumptoms and known health problems when they do visit a physician. (2) H'omen appear to have a more diffuse view of illness. They often report both mental and physical symptoms, and their physical symptoms "radiate" throughout the body rather than remain localized. (3) Both sexes confuse reproductive, digestive, and urinary symptoms because the body systems overlap. (4) Some sex differences in diagnoses for a particular symptom reflect real morbidity differences. (5) There is little evidence that women and men differ in their perception, interpretation, and description of physical symptoms. (6) The evidence for sex bias in physicians' diagnoses is scant.

KEY WORDS: sex differences; complaints; diagnoses; ambulatory care; sex bias.

\section{INTRODUCTION}

Illness can be viewed from a sociomedical perspective or a medical one. The first emphasizes how individuals perceive and respond to their

${ }^{1}$ This is a revised version of a paper presented at the American Public Health Association meetings, Los Angeles, October 1978.

${ }^{2}$ Institute for Social Research School of Public Health, The University of Michigan, Ann Arbor, Michigan 48106. 
symptoms; the second, how medical professionals diagnose and treat people's conditions. Most research opts for a single perspective when studying individual health. Studies based on health interviews favor a sociomedical approach, while those based on medical records favor a medical one.

Some intriguing questions are missed when using a single perspective. How do physicians interpret patient complaints and use them in assigning diagnoses? (For example, when patients present a headache, what kinds of diagnoses are attributed by physicians?) How do patients describe the symptoms of a medical condition? (For example, when patients are diagnosed with anemia, what kinds of symptoms did they present?) Do population groups differ in how they describe the symptoms of a particular condition? Do physicians assign different diagnoses to population groups who present the same complaint? These questions can only be answered if data contain both the patient's complaint and the physician's diagnoses, i.e., data which allow both the sociomedical and the medical perspectives of an individual's health problem.

This paper utilizes data from the National Ambulatory Medical Care Survey, which contains information about complaints and diagnoses for visits to office-based physicians in the United States. The central focus of the paper is sex differences in the description of symptoms and the assignment of diagnoses. Four questions are considered: (1) What complaints do women and men bring to physicians? (2) What diagnoses do physicans give to women and men patients? (3) When women and men present the same complaint, do physicians assign similar diagnoses to them? (4) When women and men are diagnosed with the same condition, how did they initially describe the problem to the physician? Plausible explanations will be offered for sex differences found.

\section{DATA SOURCE}

The National Ambulatory Medical Care Survey (NAMCS) is a continuous survey of visits made to office-based physicians in the United States. The survey was started in 1973 and is conducted by the National Center for Health Statistics (Tenney et al., 1974). For each sampled visit, a special Patient Record is filled out. It includes information about patient sex, patient complaints, seriousness of problem, major reason for visit (e.g., acute problem, chronic problem, examination), diagnoses, diagnostic and therapeutic services, dispositions for further care, and several other items. A copy of the 1975 Patient Record appears in a report by Koch and McLemore (1978). 
For this analysis, visits by adults (18 years or older) in 1975 are studied. In that year, 29,988 adult female visits and 16,880 adult male visits were enumerated. General results for 1975 are reported by Koch and McLemore (1978).

\section{VARIABLES}

The Patient Record variables used in this analysis are: complaints, diagnoses, sex, seriousness, and main reason for visit.

Physicians checked a box to indicate how serious the patient's principal problem was (very serious, serious, slightly serious, not serious). They checked one or more boxes to indicate the main reason for the visit (acute problem, acute problem follow-up, chronic problem-routine, chronic problem-flare-up, prenatal care, postnatal care, postoperative care, well-adult/child exam, family planning, counseling/advice, immunization, referred by other physician/agency, administrative purpose, other).

Some explanation of the complaint and diagnosis variables is necessary. The Patient Record allows three complaints and three diagnoses to be listed. Physicians enter the complaints ("in patient's own words") at the beginning of the visit. Later they write in their diagnoses and other information. Physicians are instructed to write the principal complaint and principal diagnosis first in each section, followed by those of lesser importance.

In data processing, complaints are coded according to the Symptom Classification developed for NAMCS (Meads and McLemore, 1974): ${ }^{3}$ Diagnoses are coded according to the International Classification of Diseases (ICDA) (National Center for Health Statistics, 1967). Specific complaints and diagnoses have a three-digit code. These are often aggregated into general groups of complaints and diagnoses. The general groups for this analysis are the ones used by each classification scheme, with minor exceptions. ${ }^{4}$

Several mnemonics are used in this article: GC is general complaint, GD is general diagnosis, $\mathrm{SC}$ is specific complaint, and SD is specific

\footnotetext{
${ }^{3}$ This classification has been revised and is now called the Reason For Visit Classification (Schneider et al., 1979). The revision has been used for 1978 NAMCS records and subsequent years.

4The exceptions: The reproductive symptoms here combine two major NAMCS groups (Male Reproductive System and Female Reproductive System). Examples of reproductive symptoms are cramps, pelvic pain, and swollen testes. The congenital anomalies diagnoses combine two major ICDA groups (Congenital Anomalies and Certain Causes of Perinatal Morbidity and Mortality). For adults, the second group has no cases, since it refers only to infants.
} 
diagnosis. A number following the letters signifies the mention-number (e.g., GD1 is the first diagnosis listed on the Patient Record).

For some analyses, complaints are split into two groups: symptomatic visits and nonsymptomatic vists. Examples of symptomatic visits are "headache" and "eyes watering." Examples of nonsymptomatic ones are physical exam, visit for medication, and surgical aftercare. Diagnoses are also split into two groups: sick visits and not-sick visits. Sick visits have a diagnosis of illness or injury, for example, anemia or cystitis. Examples of not-sick visits are medical exam, prenatal care, and fitting of prosthetic device.

In the text, the terms complaint and symptom are synonymous. Diagnosis and condition are also interchangeable. The term problem is used very generally, and it refers to either complaints or diagnoses. ${ }^{5}$

The analysis focuses on principal (first-mention) complaints and diagnoses. Unless otherwise noted, results refer to first-mention data.

\section{ANALYSIS PROCEDURE}

The analysis uses cross-tabulations by sex, with additional control variables. Data are weighted to correct for disproportionate sampling of visits (Tenney et al., 1974). Chi-square tests are not appropriate for weighted data; therefore, differences in distributions for women and men are not tested for statistical significance. ${ }^{6}$ The importance of sex differences is determined by (1) the size of differences (women-men) and ratios (women/men), (2) the index of dissimilarity ( $d$ ), and (3) the persistence of sex differences across categories of a control variable. ${ }^{7}$ In the text, when percentages are reported in parentheses, women's value appears first and men's second. In the tables, Patient Records with N.A. (no information given for an item) are excluded.

\section{RESULTS}

Results are presented for the four questions in the Introduction. Key results appear in italics.

\footnotetext{
${ }^{5}$ Terminology differs in the report by Koch and McLemore (1978). There, symptomatic visits are called "visits for morbidity-related problems." Patient complaints of all types are called "problems." (Thus, diagnoses are not included in that term.)

${ }^{6}$ Chi-square tests for weighted data are being developed but are not generally available yet. ${ }^{7}$ The index of dissimilarity $(\delta)$ compares two percentage distributions by the following formula: $\sum\left|x_{1, j}-x_{2, j}\right| / 2$, where $x_{1}$ and $x_{2}$ are two population groups, and $j$ is for categories of the distributed variable. The index ranges from 0 (no difference in distributions) to 100 . It is interpreted as the percentage of one group which would have to change categories in order to match the other group's distribution. For this analysis, $x_{1}$ and $x_{2}$ are women and men.
} 


\section{Sex Differences in Complaints}

Table I shows the complaints presented by women and men. For both sexes, a substantial number of visits are for nonsymptomatic reasons (35.1\% for women, $27.0 \%$ for men). When sex-specific complaints are excluded, the percentages of nonsymptomatic visits are almost equal (28.8, $27.5 \%$ ). But the types of nonsymptomatic visits differ by sex: Women are more likely to come for medication and advice ("other visit"), and men for an examination.

Women and men are therefore equally likely to report a symptom of illness or injury to physicians. The complaints (GC1) they report differ slightly $(\delta=8.0)$ : Women are more likely to complain of general, nervous, and mental symptoms. Men bring more musculoskeletal, skin/hair, and respiratory symptoms. Second and third complaints show similar small differences.

Looking at the 25 most frequent specific complaints (SC1) for each sex, there are many similarities (Table II). For example, of the 5 leading complaints for each sex, 3 are the same (lower extremity pain, progress visit, surgical aftercare). But the lists also show some notable sex differences. Nonsymptomatic visits tend to rank higher for women than men, and they account for a larger share of women's visits $(28.0,24.1 \%)$. Fatigue, headache, and abdominal pain rank higher for women than for men (although the percentages do not differ much). Several complaints appear in the top-25 list for women but not for men: weight gain, vision dysfunction, and depression. What about men? For them pains in the back/extremities/ chest rank higher and account for more visits $(10.2,17.1 \%)$. Several complaints appear in men's top-25 list but not in women's: required physical exam, skin wound, shortness of breath, and anus-rectum symptoms.

Overall, women and men report similar number of complaints $\delta^{\delta}=$ 1.2) (Table III). This hides an intriguing difference: Symptomatic women report more complaints than comparable men. But among nonsymptomatic people, men report more. This suggests that (a) women elaborate more about their problems and (b) men often initially say they have come for an examination or tests and then mention some physical symptoms.

Women report a greater variety of illnesses and injuries than men do. The top-5 illness/injury symptoms comprise only $13.7 \%$ of women's visits, compared to $19.7 \%$ for men. For the top- 10 symptoms, the percentages are 22.7 and $29.7 \%$. Thus, men's visits are somewhat more limited to particular symptoms than women's are.

Women report a combination of physical and mental symptoms more readily than men do. When they have more than one complaint, women are more likely to present a mix of physical and mental symptoms (Table IV). Men tend to bring in only-physical or only-mental complaints. 


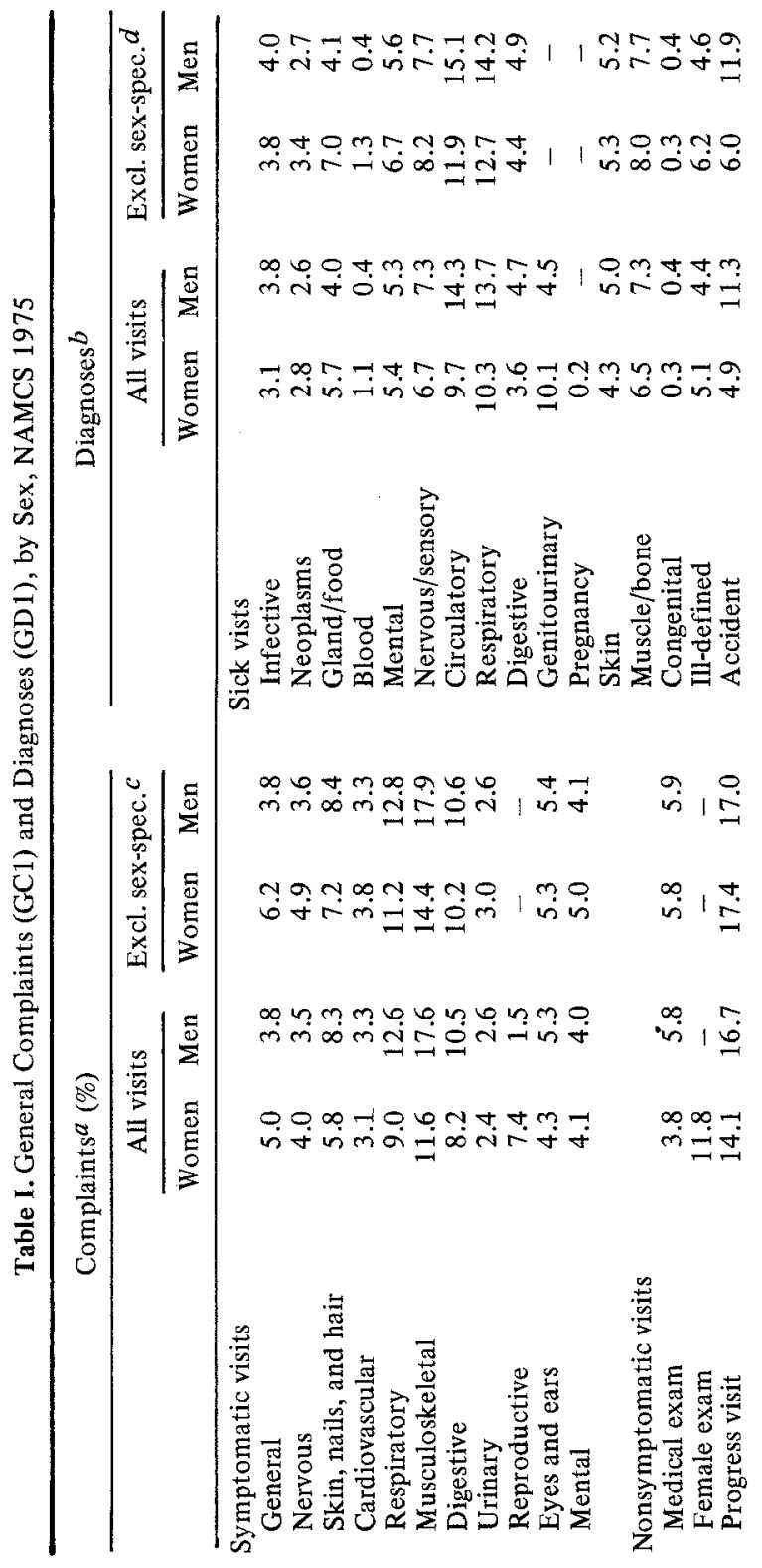




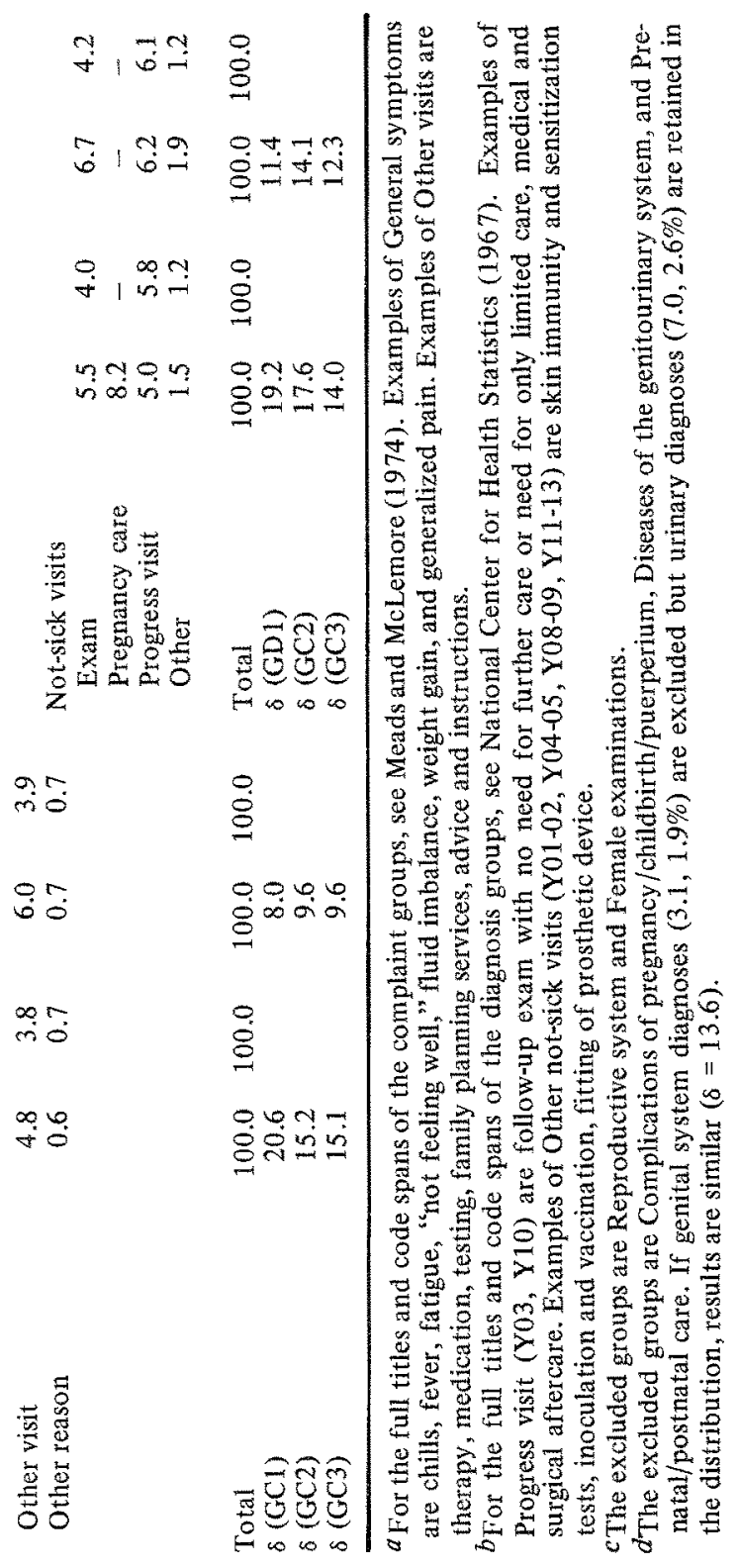




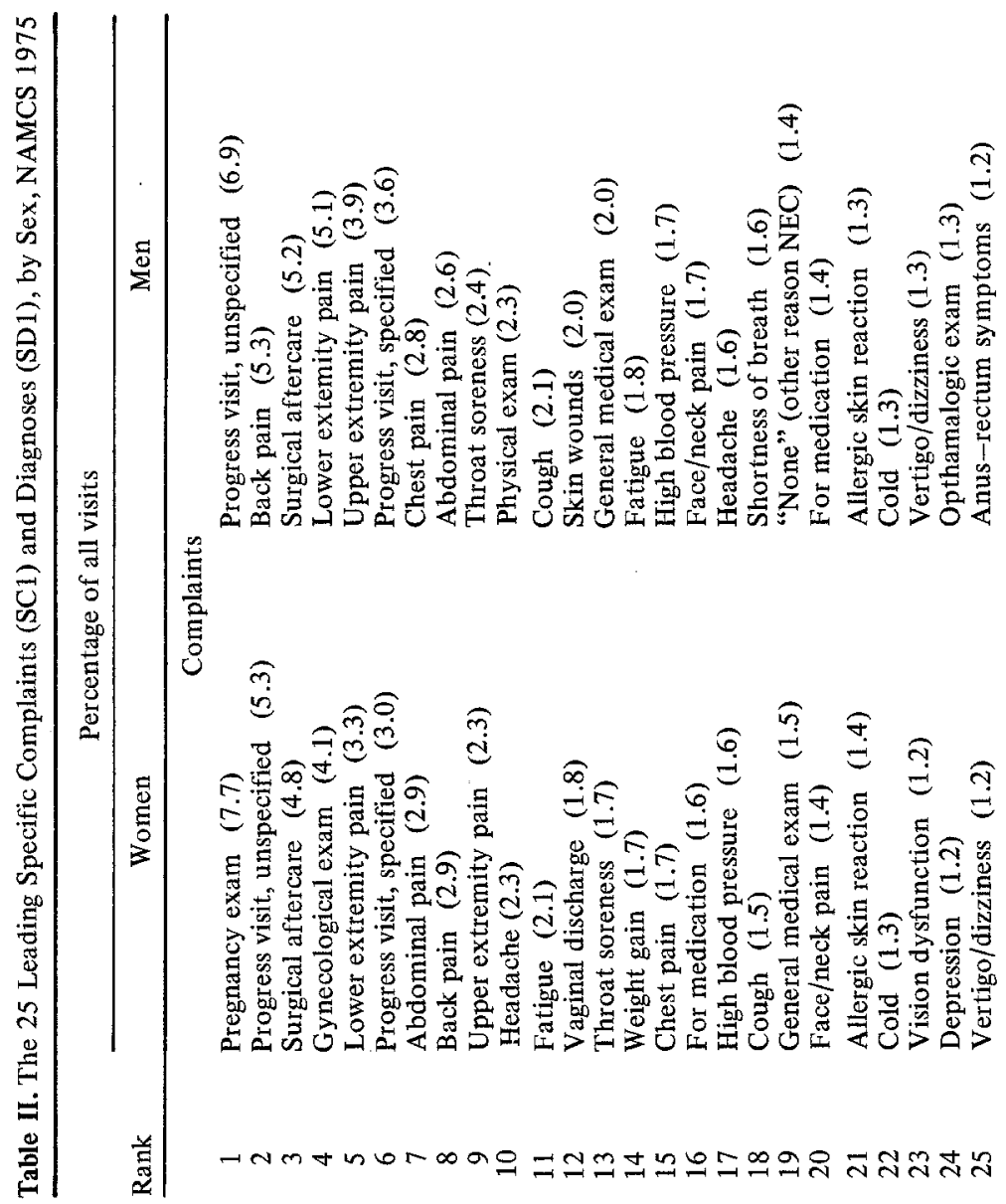




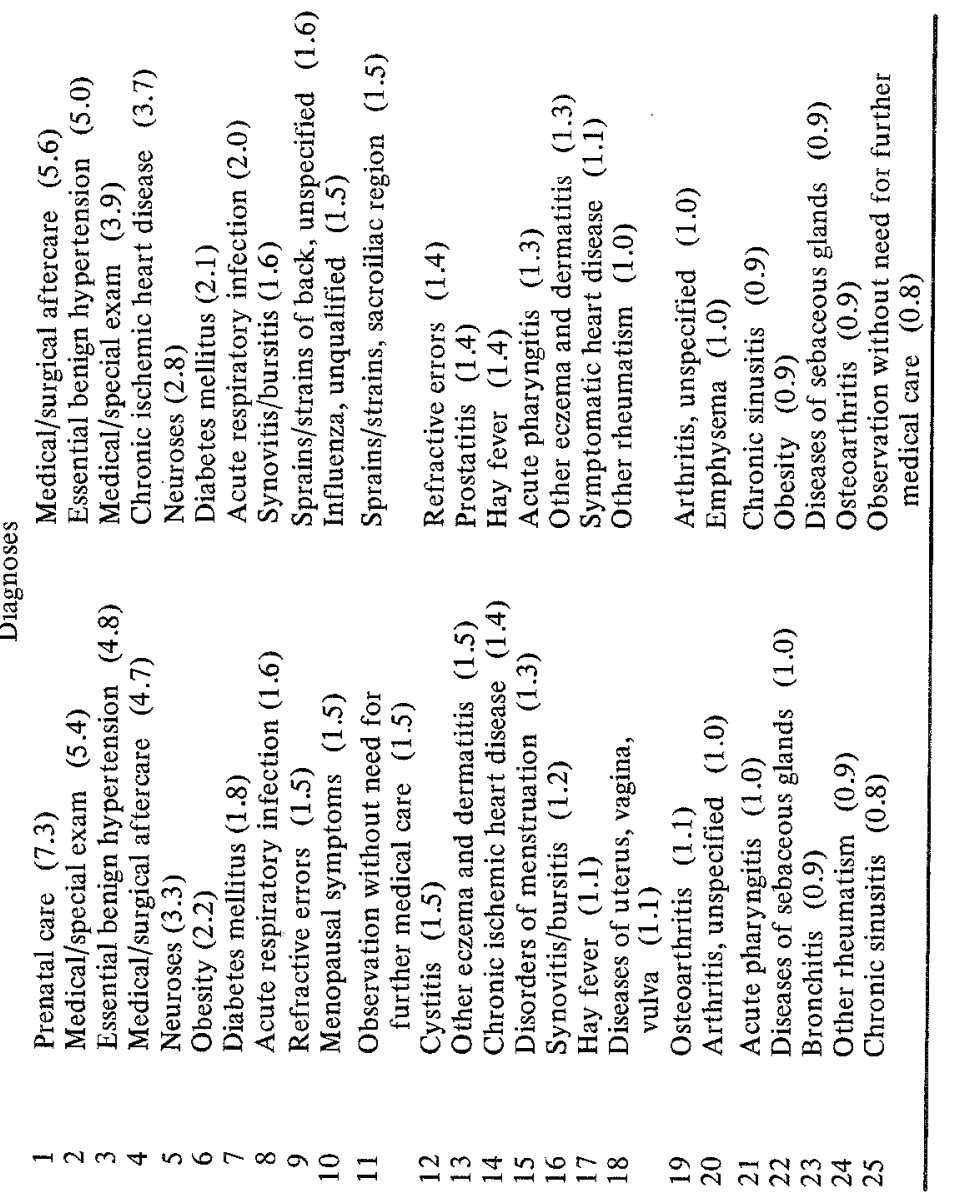


Table III. Number of Complaints and Diagnoses, by Sex, NAMCS $1975^{\circ}$

\begin{tabular}{|c|c|c|c|}
\hline \multirow[b]{2}{*}{ Symptom status } & \multicolumn{3}{|c|}{ Number of complaints } \\
\hline & 1 & 2 & 3 \\
\hline \multicolumn{4}{|l|}{ Total visits } \\
\hline Women & 69.3 & 27.3 & 3.4 \\
\hline Men & 70.5 & 26.4 & 3.1 \\
\hline \multicolumn{4}{|l|}{ Symptomatic visits } \\
\hline Women & 66.1 & 29.9 & 4.0 \\
\hline Men & 71.2 & 25.5 & 3.3 \\
\hline \multicolumn{4}{|l|}{ Nonsymptomatic visits } \\
\hline Women & 74.9 & 22.6 & 2.5 \\
\hline \multirow[t]{2}{*}{ Men } & 68.0 & 29.0 & 3.0 \\
\hline & \multicolumn{3}{|c|}{ Number of diagnoses } \\
\hline Sick status & 1 & 2 & 3 \\
\hline \multicolumn{4}{|l|}{ Total visits } \\
\hline Women & 64.6 & 30.4 & 5.0 \\
\hline Men & 64.5 & 29.8 & 5.7 \\
\hline \multicolumn{4}{|l|}{ Sick visits } \\
\hline Women & 60.8 & 33.4 & 5.8 \\
\hline Men & 63.4 & 30.6 & 6.0 \\
\hline \multicolumn{4}{|l|}{ Not-sick visits } \\
\hline Women & 79.6 & 18.2 & 2.2 \\
\hline \multirow[t]{2}{*}{ Men } & 73.8 & 23.1 & 3.1 \\
\hline & \multicolumn{3}{|c|}{ Number of diagnoses } \\
\hline Number of complaints & 1 & 2 & 3 \\
\hline \multicolumn{4}{|l|}{1} \\
\hline Women & 74.9 & 22.4 & 2.7 \\
\hline Men & 73.8 & 22.8 & 3.4 \\
\hline \multicolumn{4}{|l|}{2} \\
\hline Women & 42.9 & 48.4 & 8.7 \\
\hline Men & 44.7 & 46.0 & 9.3 \\
\hline \multicolumn{4}{|l|}{3} \\
\hline Women & 27.4 & 51.0 & $21.6^{b}$ \\
\hline Men & 21.7 & 49.2 & 29.1 \\
\hline
\end{tabular}

$a$ In percentages. Rows add to $100 \%$.

$b^{\text {This }}$ category represents less than $1 \%$ of women's visits and less than $1 \%$ of men's.

\section{Sex Differences in Diagnoses}

Table I shows the diagnoses given to women and men. Women are more likely to receive a "not-sick" diagnosis $(20.2,11.0 \%)$. But this includes visits for pregnancy care. When sex-specific diagnoses are excluded, the percentages of not-sick visits are more similar $(14.8,11.5 \%)$. The difference is mainly because women make more visits for examinations than men do. 
Table IV. Mixing Mental and Physical Complaints and Diagnoses, by Sex, NAMCS $1975^{a}$

\begin{tabular}{|c|c|c|c|c|c|c|}
\hline \multicolumn{7}{|c|}{ Mixing mental and physical complaints } \\
\hline & \multicolumn{3}{|c|}{$\mathrm{GC} 2$} & \multicolumn{3}{|c|}{$\mathrm{GC} 3$} \\
\hline & Mental & Physical & Other & Mental & Physical & Other \\
\hline \multicolumn{7}{|l|}{$\mathrm{GC} 1$} \\
\hline \multicolumn{7}{|l|}{ Mental } \\
\hline Women & 37.0 & 41.7 & 21.3 & 36.1 & 46.1 & 17.8 \\
\hline Men & 44.5 & 33.0 & 22.5 & 57.9 & 23.7 & 18.5 \\
\hline \multicolumn{7}{|l|}{ Physical } \\
\hline Women & 3.0 & 89.4 & 7.6 & 5.9 & 78.1 & 16.0 \\
\hline Men & 2.2 & 92.1 & 5.7 & 3.1 & 82.8 & 14.1 \\
\hline \multicolumn{7}{|c|}{ Mixing mental and physical diagnoses } \\
\hline & \multicolumn{3}{|c|}{ GD2 } & \multicolumn{3}{|c|}{ GD3 } \\
\hline & Mental & Physical & Other & Mental & Physical & Other \\
\hline \multicolumn{7}{|l|}{ GD1 } \\
\hline \multicolumn{7}{|l|}{ Mental } \\
\hline Women & 44.5 & 55.0 & 0.5 & 30.2 & 65.2 & 4.6 \\
\hline Men & 56.3 & 42.4 & 1.3 & 45.0 & 53.7 & 1.3 \\
\hline \multicolumn{7}{|l|}{ Physical } \\
\hline Women & 3.9 & 89.5 & 6.6 & 7.2 & 86.0 & 6.8 \\
\hline Men & 2.4 & 93.2 & 4.4 & 5.6 & 90.0 & 4.4 \\
\hline
\end{tabular}

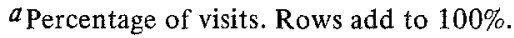

Women and men are therefore about equally likely to be diagnosed with an illness or injury ("sick'). The diagnoses (GD1) differ slightly ( $\delta=$ 11.4): Women receive substantially more diagnoses of gland/food and illdefined conditions, and slightly more of blood, mental, and neoplasm conditions. Men receive notably more diagnoses of circulatory conditions and accidents and, to a lesser extent, respiratory and digestive conditions. Second and third diagnoses show similar sex differences.

Looking at the 25 most frequent specific diagnoses (SD1), one similarity stands out (Table II). Four of the five leading diagnoses are the same for both sexes (medical/special exam, medical/surgical aftercare, essential benign hypertension, neuroses). Otherwise, there are striking differences in specific diagnoses: For women, not-sick titles take a larger share of all visits (17.4\%including prenatal care, but $10.1 \%$ excluding it; $9.5 \%$ for men). Two titles rank much higher for women (although the percentages do not differ much): obesity and "observation of a condition without need for further care". Two diagnoses appear on women's list

" "Observation without need for further medical care" (ICDA 793) is a "sick" diagnosis, even though the visit is largely administrative. Instructions for this code are: "Cases which present some symptoms or evidence of an abnormal condition which requires further study, but which after examination and observation show no need for further treatment or medical care" (National Center for Health Statistics, 1967). 
only: cystitis and bronchitis. For men, these conditions rank higher and account for more visits than for women: heart disease $(1.4,4.8 \%)$, respiratory conditions $(5.4,8.1 \%)$, and musculoskeletal problems $(1.2$, $4.7 \%$ ). Several diagnoses appear only on men's list: symptomatic heart disease, influenza, emphysema, sprains/strains of back, and sprains/strains of sacroiliac region.

Two other variables offer diagnostic information: major reason for visit and seriousness of problem.

On the Patient Record, physicians summarize the main reason(s) for the visit. Women and men's visits are equally likely to be for chronic problems (28.2 and $28.5 \%$ for "routine" chronic problem; 11.8 and $11.7 \%$ for flare-up of chronic problem). But men's are more often for acute problems (26.4 and $32.0 \%$ for acute problem; 11.6 and $15.2 \%$ for acute problem follow-up). This is compatible with men's higher percentage of respiratory and accident diagnoses, noted above. More of women's visits are for nonsymptomatic reasons (well-adult exam, family planning, counseling or advice).

According to physicians, men's conditions are more serious than women's. For men, $24.7 \%$ of their problems are "very serious" or "serious," compared to $17.6 \%$ for women. These conditions (GD1) are particularly serious for men compared to women: neoplasms, diseases of blood and blood-forming organs, mental disorders, and genitourinary conditions. Specific conditions (SD1) which are more serious for men are influenza, neuroses, osteoarthritis, and back sprains/strains. Only a few conditions are more serious for women: diabetes, bronchitis, arthritis, and synovitis/bursitis.

Overall, women and men receive similar numbers of diagnoses $(\delta=$ 0.7) (Table III). But when sick status is controlled, an interesting sex difference appears: Not-sick men receive more diagnoses than comparable women. This suggests that more problems are found during examination visits for men than for women. By contrast, sick women receive slightly more diagnoses than sick men, but the difference is small.

Women receive a slightly greater variety of diagnoses, but the difference is small. The top-5 sick diagnoses comprise $13.7 \%$ of women's visits, compared to $15.6 \%$ for men. Similarly, the top-10 sick diagnoses encompass $21.2 \%$ of women's visits and $23.2 \%$ of men's.

Physicians give a mix of physical and mental diagnoses to women more than to men (Table IV).

\section{Sex Differences in Diagnoses Given to Complaints}

How do physicians evaluate information provided by patients to arrive at diagnoses? Are symptomatic women and men equally likely to be 
diagnosed as sick? For a particular complaint, what diagnoses do physicians give to them? Does one sex receive more diagnoses for their complaints than the other?

Virtually all patients who complain about a symptom are diagnosed as sick $(97.0,97.4 \%)$ (Table V). Physicians may feel it is safer to give a sick diagnosis to a symptomatic patient than not to (cf. Freidson, 1973; Scheff, 1966).

The remaining $3 \%$ of visits are for patients who complain of a symptom but are diagnosed as not sick. Not-sick status is assigned to some symptoms more than others (Table V), and some sex differences appear. For reproductive complaints, women are considered not-sick more than comparable men $(9.1,3.0 \%)$. To a lesser extent, this also occurs for digestive, general, and mental complaints. Men are more often diagnosed as not-sick for their urinary and eye/ear complaints and, to a lesser extent, for skin/hair and musculoskeletal ones.

A sizable percentage of patients who say they are nonsymptomatic end up with a sick diagnosis ( $48.1 \%$ for women, $66.9 \%$ for men) (Table V). Excluding female exams, the sex difference is reduced $(57.5 \%, 66.9 \%)$ but nonsymptomatic men are still more likely to be sick. This occurs when men come for progress visits, "other visits" (therapy, medication, advice), and "other reason" visits. But there is an anomaly: Women who come for a medical examination are more often diagnosed as sick than comparable men $(55.2,41.5 \%)$. This also occcurs when women come specifically for medication only ( $\mathrm{SC} 1)(70.7,54.5 \%)$. An explanation will be offered later.

At the bottom of Table $\mathrm{V}$, the overall distribution of visits by symptom and sick status is shown. A larger percentage of all women's visits is "nonsymptomatic and not sick"; these are visits for exams, medication advice, etc. More of men's visits are "symptomatic and sick." The sexes are equally likely to be "symptomatic but not sick"- a situation often labeled as hypochondria.

Sometimes physicians think that a patient is ill, but they have trouble identifying the problem and they resort to an "ill-defined" diagnosis (ICDA 780-796). As noted before, women are more likely than men to receive such diagnoses. The sex difference is especially large for digestive and mental complaints and for examination visits (Table V). This is curious; it is difficult to see why such complaints are harder to diagnose for women patients, and why their examinations lead to less certain conclusions.

Sometimes physicians think that a physical complaint actually reflects a mental problem, and they assign a diagnosis of mental disorder (ICDA 290-319). Women are more likely to receive this diagnosis, especially for GD2 and GD3. Mental disorder diagnoses are especially likely for women who have nervous symptoms, compared to men (Table V). It is understandable that some nervous symptoms (such as headache, tingling, insomnia) can reflect mental disorders rather than nervous disorders, but 


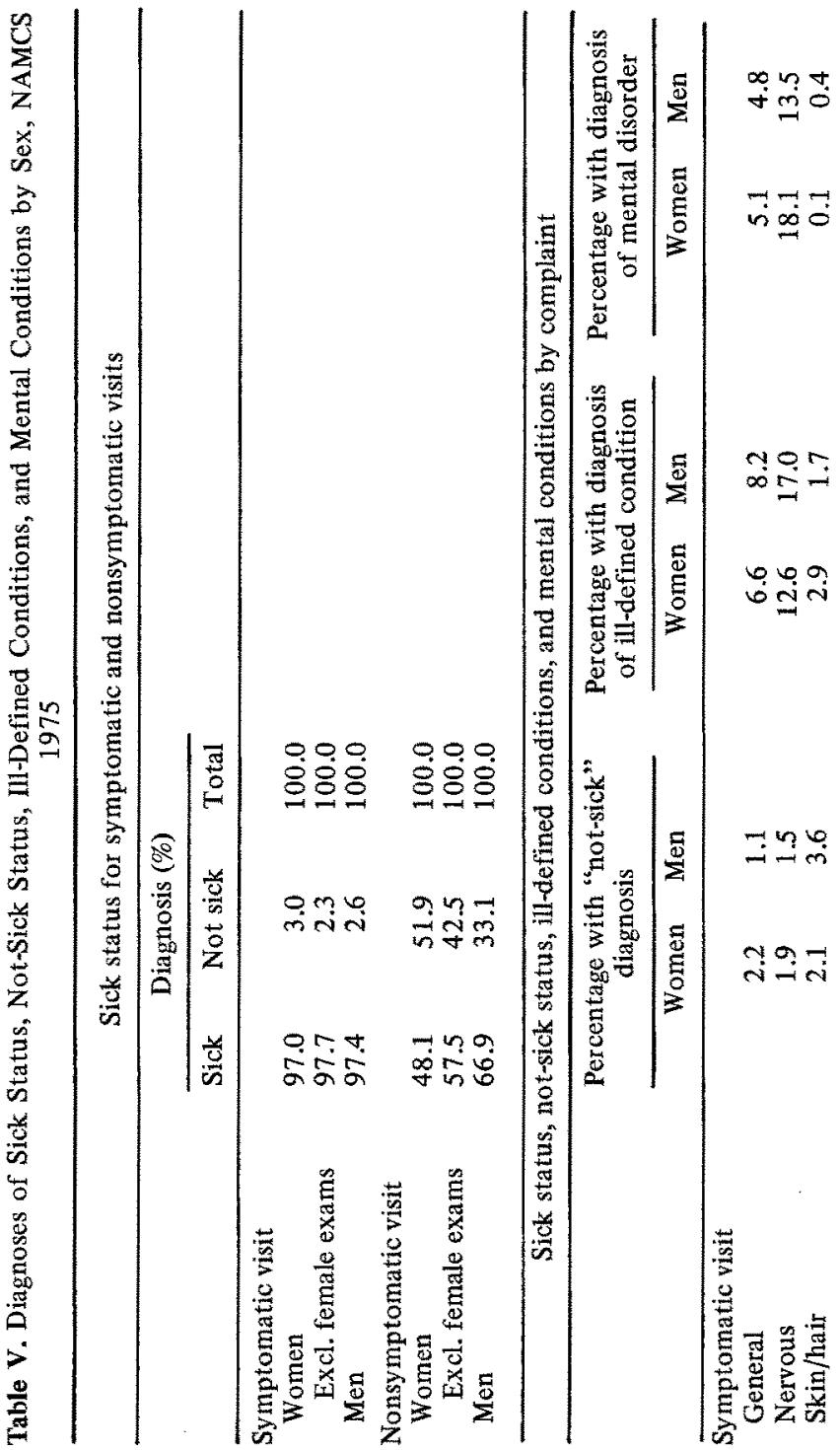




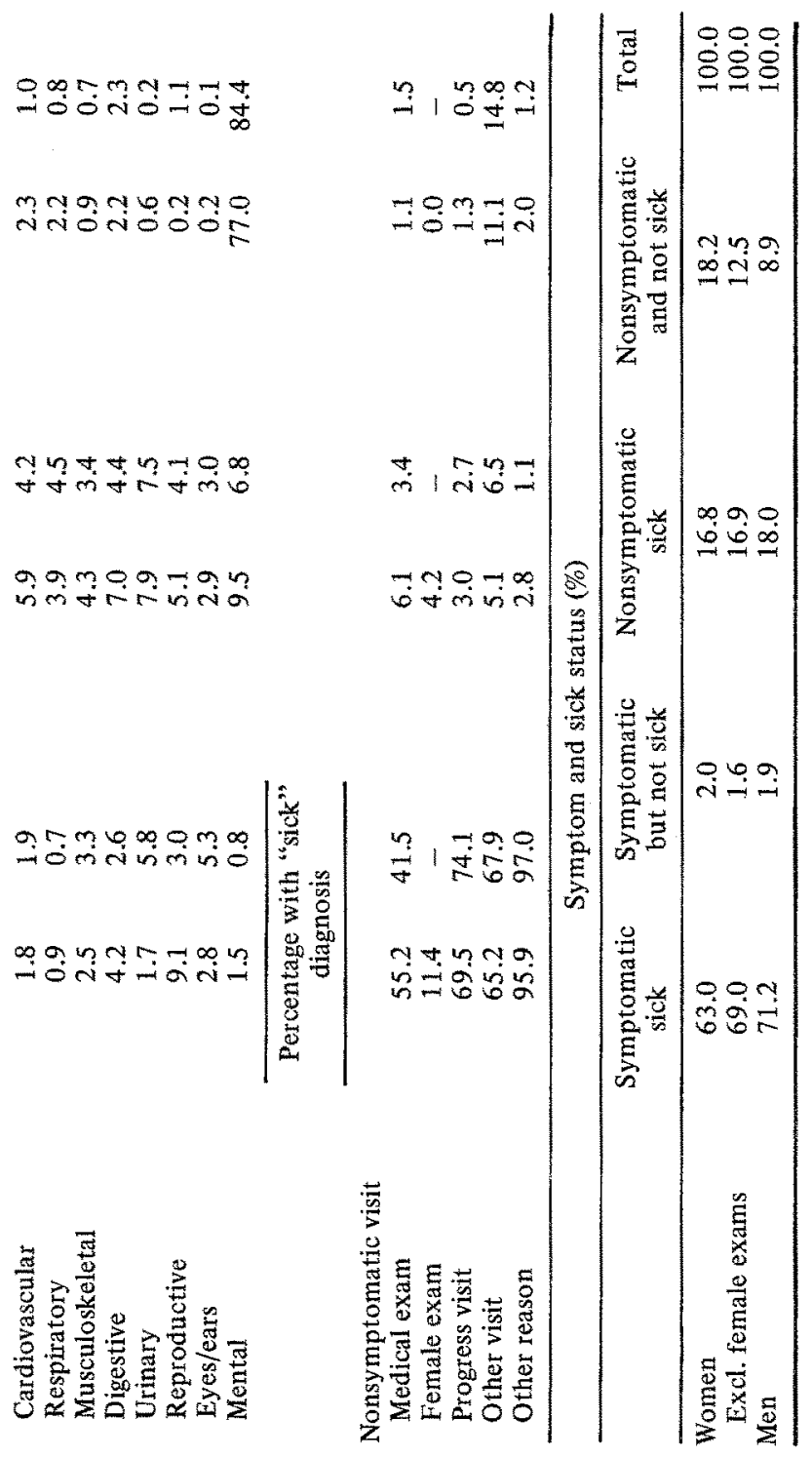


why this occurs more frequently for women than men is not clear. For two complaints $(\mathrm{GCl})$ men do receive more diagnoses of mental disorder: mental symptoms and other visits. This is intriguing; possible reasons will soon be suggested.

What diagnoses are given to particular complaints, and do these differ for women and men? Consider diagnoses given to general complaints (GC1) and to the 25 most frequent specific complaints (SC1). Only the most striking sex differences will be noted.

For general complaints (GC1), women receive more diagnoses of gland/food problems $(40.4,22.3 \%)$ and men receive more respiratory diagnoses $(10.9,20.5 \%)$. This is partly because they state different specific complaints; women report more weight gain, whereas men report more fever, fatigue, and general pain.

For nervous complaints (GC1), men receive more ill-defined diagnoses $(12.6,17.0 \%)$. It is not obvious why. Headache (SC1) is a very common nervous complaint. It leads to relatively more mental and nervous diagnoses for women (20.9 and $13.4 \%$ for mental diagnosis; 19.9 and $10.2 \%$ for nervous diagnosis). Men with headaches receive more diagnoses of respiratory conditions $(14.7,26.9 \%)$.

Men's visits for skin/hair, musculoskeletal, and eye/ear symptoms (GC1) are diagnosed as accidents more often than for women $(9.6$ and $23.6 \%$ for skin/hair; 24.8 and $37.2 \%$ for musculoskeletal; 3.1 and $10.6 \%$ for eye/ear). This reflects men's higher injury rates (Drury, 1977). Specific complaints (SC1) of back pain, lower extremity pain, and upper extremity pain are diagnosed as muscle/bone problems more often for women than men ( 41.5 and $31.9,36.5$ and $28.8,49.5$ and $44.1 \%$, respectively). This partly reflects women's higher prevalence of arthritis.

Men receive more diagnoses of circulatory conditions for virtually all complaints (GC1), including nonsymptomatic visits. The sex difference is especially large for specific complaints (SC1) of fatigue, chest pain, weight gain, and vertigo (18.8 and 27.0,34.9 and 48.8, 3.8 and 11.6, 36.5 and $41.2 \%$, respectively).

Women with a digestive complaint $(G C 1)$ receive more diagnoses of urinary and genital/uterine conditions than comparable men ( 2.1 and $0.8 \%$ for urinary; 4.0 and $1.5 \%$ for genital/uterine). This is especially true for the specific complaint (SC1) of abdominal pain (15.8 and 6.0\% total for genitourinary diagnoses). Men with a urinary complaint $(G C 1)$ receive more genital diagnoses $(5.9,31.5 \%)$, and vice versa for genital complaints $(1.3$, $7.2 \%$ ). Apparently, women have more trouble identifying the source of abdominal discomforts, and men confuse urinary and genital problems.

Women who come for other visits (GC1) receive more diagnoses of gland/food and genitorinary conditions than men do (14.1 and $8.7 \%$ for gland/food; 11.2 and $1.7 \%$ for genitourinary). This is especially true when 
women come for medication ( $\mathrm{SC} 1)$. The sex difference is partly because more women come specifically for diet control and family planning and therefore receive diagnoses of obesity, urinary infection, etc. A similar result: Women coming for medical exams (GC1) receive nervous/sensory diagnoses more than men $(26.8,17.6 \%)$. This is partly because more of women's visits are specifically for eye exams.

How often do diagnoses concur with complaints (e.g., a digestive complaint is diagnosed as a digestive condition)? Are there sex differences in the degree of agreement between patient and physician? For this analysis, the most likely diagnosis (GD1) was selected for each general complaint (GC1). Then the percentages of complaints which received the "appropriate" diagnosis were compared for women and men. Overall, the degree of agreement is very similar for the sexes. In other words, physicians tend to concur with the presenting complaint about as often for women as men (data not shown). There are two interesting exceptions: Physicians agree more with men who present mental and reproductive symptoms than with comparable women (77.0 and $84.4 \%$ for mental; 57.8 and $68.7 \%$ for reproductive). An explanation for this intriguing result will be of fered soon.

Women and men receive the same number of diagnoses per complaint (Table II). If there is any difference at all, men appear to receive slightly more diagnoses per complaint then women do.

\section{Sex Differences in Complaints Underlying Diagnoses}

Let us reverse the perspective and ask about complaints presented for particular diagnoses. How do women and men differ in their presenting complaints when they receive the same diagnosis?

Consider the complaints behind general diagnoses (GD1) and the 25 most frequent specific diagnoses (SD1). The most striking sex differences are reported here.

When diagnosed with infections, neoplasms, and genitourinary conditions (GD1), more women had a reproductive complaint (22.8 and $4.1 \%$ for infections; 22.4 and $2.7 \%$ for neoplasms; 42.7 and $24.3 \%$ for genitourinary). This may partly reflect women's higher risk of reproductive diseases and disorders.

When diagnosed with a gland/food condition (GD1), more women than men reported general symptoms $(35.5,21.3 \%)$. Men tend to report more musculoskeletal symptoms $(5.0,12.2 \%)$ or say they came for a

${ }^{9}$ For some results in this section, tables from the previous section are percentaged in the opposite direction and used. Repercentaged tables do not contain new information, but they can show results hidden by the first perspective. In this section, only new results are reported, omitting any that are redundant with the previous section. 
progress visit $(29.6,40.1 \%)$. The data partly reflect different specific ailments: Obesity and myxedema (a thyroid condition) diagnoses are more common for women, and diabetes for men.

For diagnoses of blood conditions (GD1), women tend to report more general symptoms $(37.2,31.6 \%)$. Men complain of digestive troubles (1.9, $12.2 \%$ ) or have come for a progress visit $(13.8,21.6 \%)$. Most of the blood condition diagnoses are for anemia, which is more prevalent among women in the general population.

For the majority of specific illness and injury titles (SDI), women present a greater variety of symptoms than men do. This is especially true for cystitis (a bladder condition). Men with cystitis usually report a urinary symptom, but many women report general, digestive, and reproductive symptoms.

For essential benign hypertension and ischemic heart disease (SDI), more men say they came for a progress visit $(38.7$ and $46.9 \%$ for hypertension; 31.4 and $42.2 \%$ for ischemic heart disease). A possible explanation will be stated soon.

\section{EXPLAINING SEX DIFFERENCES IN COMPLAINTS AND DIAGNOSES}

During a medical visit, a physician tries to determine if a patient is "sick" (ill or injured) or "not sick." If the patient is "sick,"' the physician strives to diagnose the ailment and recommend appropriate treatment. A physician's behavior is influenced by medical skills, personal attitudes, and treatment preferences. The patient also contributes attitudes, motivations, and information to the encounter. Thus, a large number of social-psychological and medical factors affect the outcome of a visit. Some originate with the physician, some with the patient.

What are possible explanations for male-female differences in complaints and diagnoses? Consider first factors that may produce sex differences in the designation sick vs. not sick. Then consider factors that influence the specific diagnosis assigned.

\section{Sick Status}

If women and men differ in receiving a sick diagnosis, there are four possible explanations. Three refer to patient motivations which influence whether a medical visit occurs and when it occurs; one refers to physician motivations.

(I) Ignorance of Conditions. People may have an asymptomatic condition which has not been diagnosed. They are entirely unaware of the 
problem. Because women visit physicians more frequently than men do (Drury, 1977), they probably get earlier diagnoses of chronic conditions. When men visit, they may often consider themselves healthy and, to their surprise, be diagnosed as sick,

(2) Denial of Perceived Symptoms or of Diagnosed Conditions. People may feel a symptom or even know about a condition from prior diagnosis, but still be reluctant to admit that it exists. Popular belief holds that men tend to deny symptoms more than women do. If so, men are less likely to seek medical care, and even when they seek it, they may not report a problem they know about. Again, this can lead to more sick diagnoses among men who claim to be in good health.

(3) Symptom Perception and Interpretation. People vary in their threshold of physical discomfort and also in whether a particular discomfort is taken as a sign of illness. It is widely believed that women have a lower threshold for perceiving discomforts, but there is no scientific evidence on the issue.$^{10}$ It is also thought that women have a broader interpretation of illness than men. Symptom interpretation has received some attention (Apple, 1960; Levine and Kozloff, 1978; Mechanic, 1972). Suchman (1965a) reports some sex differences in symptom interpretation, but the results are flawed because respondents discussed their interpretations long after the illness events.

What happens if women are more sensitive to body discomforts and more likely to think a discomfort means illness? More than men, they may bring minor discomforts to a physician, who finds no medical indication of illness and gives a not-sick diagnosis.

(4) Physician Sex Bias. Sex bias occurs when a physician offers diagnoses and treatment on the basis of a patient's sex, assuming certain problems and needs for men and different ones for women. Some people believe that women are diagnosed and treated in a more cavalier fashion than men, i.e., that medical criteria are less important in determining visit outcomes for women. They claim that women are more likely to be told they are not sick when presenting a complaint, to be diagnosed as sick without medical indications, to receive a psychogenic diagnosis, and to receive unnecessary services and advice for follow-up care. These claims are discussed by Ehrenreich (1974), Fidell (1980), Howell (1974), and Lennane and Lennane (1973).

There is little research evidence on physician sex bias. Armitage et al. (1979) find that men receive a more extensive workup than women for several common complaints. McCranie et al. (1978) conducted an experiment in which physicians were presented with identical profiles for

\footnotetext{
${ }^{10}$ Sex differences in pain perception have been studied (Notermans and Tophoff, 1967; Woodrow et al., 1972), but not sex differences in illness perception.
} 
female and male patients. They find no evidence that physicians diagnose women with more psychogenic illness than men. Wallen et al. (1979) find that physicians provide more explanations to women during a visit, but apparently briefer ones than for men. Also, physicians' explanations are less likely to match the level of technicality of women's questions than of men's.

Sex bias can take two forms when assigning sick or not-sick status. First, physicians may say women are sick without good clinical evidence or any reported symptoms more than for men. Second, they may belittle women's complaints and fail to diagnose an illness (i.e., assign not-sick status).

\section{Diagnoses}

If the sexes differ in the diagnoses received for particular complaints, or if they differ in the complaints which are stated for particular diagnoses, there are five possible explanations. Four refer to social-psychological or medical characteristics of patients; one refers to physician motivations.

(1) Diffuse View of Illness. People probably differ in how local or general they feel symptoms are, and in how closely related physical troubles and emotional upsets are. For some, physical symptoms radiate so that discomforts seem to be in many places rather than a few. For some, physical and mental symptoms are often companions. When visiting physicians, people with a "diffuse" view of illness may report symptoms in many places, or they may report a combination of physical and mental discomforts. In such cases, physicians may have trouble interpreting the information and finding the principal problem. There is no prior research to indicate how women and men differ in these two factors. Popular opinion suggests that women have a more diffuse view, so they tend to "feel bad all over" and to be depressed when ill.

(2) Symptom Description. Virtually nothing is known about how people describe symptoms-how much they elaborate, whether they report general or specific symptoms, how they use medical or lay terms, etc. There are some hints that women and men differ in their modes of describing physical problems: Wallen et al. (1979) tape-recorded physician-patient encounters and found that women ask more questions during the visit. Further analyses are planned to see if women also spend more time informing the physicians about their physical problems. Women tend to be more informed about health, especially about symptoms that are clues for serious conditions (Feldman, 1966; Suchman, 1965b). This may expand their "health vocabulary" so they can talk about symptoms at greater length and in more detail. In addition, psychological research consistently shows that women are more skilled verbally than men (Maccoby and 
Jacklin, 1974). Overall, women may be able to elaborate their troubles by reporting more symptoms and more specific ones than men. Better description could lead to higher concurrence of the ultimate diagnosis with women's presenting complaints than with men's.

(3) Confusion of Body Systems. When two body systems overlap a good deal, symptoms in one can be confused with the other. Women's reproductive, digestive, and urinary systems overlap greatly. Men's reproductive and urinary systems also overlap. This can cause confusion when women try to identify the site of abdominal discomforts, and when men have symptoms in their urinary/genital area. When diagnosing symptoms, physicians must correct these confusions.

(4) Different Conditions for the Same Symptom. There is no one-to-one correspondence between a symptom and a condition. In other words, a particular symptom (such as headache) is an indication of various conditions. In the general population, women and men differ in prevalence rates for many acute and chronic conditions (Verbrugge, 1981). Thus, a given symptom can often result in different diagnoses for them.

(5) Physician Sex Bias. If physicians opt for specific diagnoses because of stereotyped views about the sorts of troubles women and men have, this is sex bias. When different diagnoses are given to women and men with the same complaint, physician sex bias may be a factor.

\section{Summary}

In summary, different complaints and diagnoses for women and men patients can reflect their different health conditions, attitudes, and perceptions. They can also be due to physician sex bias. When interpreting results which show sex differences, all of the explanations must be considered.

\section{DISCUSSION}

What are the most plausible explanations for the sex differences found in NAMCS data? The following explanations are tentative and offered for further discussion and testing; the NAMCS data cannot test them directly.

\section{Ignorance and Denial}

Some results suggest that men patients are unaware of asymptomatic conditions and that they are reluctant to admit perceived symptoms and even some previously diagnosed conditions: 
More men patients are "nonsymptomatic but sick" and "symptomatic and sick." The former suggests that men have more asymptomatic problems that are diagnosed during the visit or that they hesitate to admit their symptoms and conditions.

Men receive more circulatory diagnoses for virtually all kinds of complaints. This is a sign that they are unaware of heart problems or reluctant to mention them.

For "progress visits," men are more likely than women to receive diagnoses of gland/food conditions, blood diseases, hypertension, and ischemic heart disease. Often, the specific gland/food diagnosis is diabetes, and the specific blood disease is anemia. Men may be unwilling to admit symptoms for these problems, preferring to say they have come for a progress visit for some other problem. Alternatively, because they visit physicians less often than women do, men are more likely to get a new diagnosis of diabetes, anemia, hypertension, and heart disease on a particular visit.

Physicians' higher concurrence with men who complain of mental and reproductive symptoms probably reflects men's unwillingness to seek care for these problems. The men who do seek help are quite troubled or ill. Most state their problem directly, and physicians usually agree. Nevertheless, some men who receive mental diagnoses still seem reluctant to admit mental symptoms, preferring to say they have come for medication or advice ("other visit").

Men may hesitate to report genital problems. They frequently report a urinary symptom which is then diagnosed as a genital condition. The reverse (genital symptoms which get urinary diagnoses) is rare!

"Not-sick" men receive more total diagnoses than not-sick women. Although the principal reason for the visit is an examination or other not-sick reason, more health problems are uncovered during the visit for men than for women.

\section{Symptom Perception and Interpretation}

Few results suggest that women and men differ in their perception and interpretation of symptoms:

Women who complain of reproductive symptoms are more likely to be diagnosed as not sick than men. To a lesser extent, this also appears for digestive, general, and mental complaints. This may indicate a lower threshold of perception for women; they feel things that men do not feel. Or it may indicate a broader interpretation of discomforts as signs of illness; women decide they are sick, whereas men just feel uncomfortable. 
Men may know less than women about signs of circulatory conditions. This could account for more circulatory diagnoses, regardless of the initial complaint, among men. (See Ignorance and Denial for an alternative explanation.)

\section{Diffuse View of Illness}

NAMCS has intriguing evidence that women have a more diffuse view of illness than men:

Three results suggest permeable boundaries for physical and mental problems. Women bring in a mix of physical and mental symptoms more often than men do. Also, more of women's nervous complaints are diagnosed as mental disorders. Finally, concurrence of diagnosis with complaint is lower for women's mental symptoms than men's. (See Ignorance and Denial for an alternative explanation.)

Three results suggest that women feel illness symptoms quite generally and do not pinpoint them as much as men do. First, for most diagnoses women present a greater variety of symptoms. Second, they bring in more general symptoms (GC1) such as fatigue, swelling, pain all over, and general malaise. Third, women's mental and digestive complaints result in more ill-defined diagnoses. This may reflect physicians' genuine difficulties in diagnosing these complaints. If the symptoms are not obvious and examination is inconclusive, an ill-defined diagnosis is a reasonable decision.

\section{Symptom Description}

There is only a little evidence of sex differences in symptom description:

Two results suggest that women have a larger health vocabulary. Symptomatic women report more complaints than symptomatic men do. And women patients tend to report a greater overall variety of symptoms. These could indicate a larger health vocabulary for women, so they elaboate their symptoms to physicians more than men do. (See Diffuse View of Illness for an alternative explanation.) One result seems to contradict this.Women report more "general" complaints (GC1) than men do. Presumably a broader vocabulary would lead to more specific complaints, not more general ones. This finding probably has more to do with a diffuse view of illness than symptom description.

There is no evidence in NAMCS that women describe their problems more accurately or clearly than men do. Physicians tend to given diagnoses that concur with complaints equally to both sexes-not more often to women. 


\section{Confusion of Body Systems}

Both sexes have difficulty identifying the source of urinary, genital, and digestive problems:

Women's digestive complaints result in more urinary and genital diagnoses than men's. This is especially true for abdominal pain (SC1).

A diagnosis of cystitis originates from digestive and reproductive complaints for women more than for men.

Men have more genital diagnoses for their urinary complaints and vice versa, than women do. (See Ignorance and Denial for an alternative explanation.)

\section{Different Conditions for the Same Symptom}

Many sex differences are due to differences in real morbidity. Specific complaints (SC1) sometimes lead to different diagnoses for perfectly sound medical reasons: ${ }^{11}$

Men's headaches are diagnosed as respiratory conditions more than women's.Men have higher rates of chronic bronchitis, emphysema, and asthma (Hadden, 1978; Higgins et al., 1977; Wilder, 1973). ${ }^{12}$ Headache is a common symptom of respiratory problems, and it is no surprise that it leads to different diagnoses for men and women.

"The text also shows some general complaints (GC1) leading to different diagnoses for women and men. Usually this is an artifact of aggregation; the specific complaints actually differ and, understandably, lead to different diagnoses. The main instances are listed here: (a) General complaints are often given gland/food diagnoses for women but respiratory diagnoses for men; (b) skin/hair, musculoskeletal, and eye/ear complaints are given relatively more accident diagnoses for men; and (c) "other visits" get gland/food and genitourinary diagnoses for women more than for men.

Aggregation also explains why some general diagnoses (GD1) come from different complaints. The specific diagnoses assigned actually differ. The main instances are: (a) Infection, neoplasm, and genitourinary diagnoses come from reproductive complaints for women more than men; and (b) gland/food diagnoses for women come relatively often from general symptoms.

${ }^{12}$ The three references rely on different surveys: the Tecumseh Community Study (Higgins $e t$ al., 1977), the Health and Nutrition Examination Survey (HANES) (Hadden, 1978), and the Health Interview Survey (HIS) (Wilder, 1974). They do not fully agree: Higher male rates for asthma appear at all ages in HIS but only for "some ages in Tecumseh and HANES. Higher male rates for chronic bronchitis appear at all ages in Tecumseh but only some ages in HANES and HIS.

${ }^{13}$ Two results for men are difficult to interpret by any explanation. Readers might like to consider them: (1) Men's nervous complaints receive ill-defined diagnoses more than women's $(12.6,17.0 \%)$. Do physicians tend to reserve judgment until more diagnostic procedures are done? (2) Men who complain of urinary and eye/ear problems are diagnosed as "not sick" more than comparable women (Table IV). To a lesser extent, this is true for skin/hair and musculoskeletal complaints too. 
Men's complaints of fatigue, chest pain, weight gain, and vertigo are diagnosed as circulatory conditions more than similar complaints by women. This partly reflects the generally higher prevalence of heart disease among men (Wilder, 1975).

More muscle/bone diagnoses for women's back/extremity pains reflect their higher arthritis rates (Epstein et al., 1965; Hadden, 1978; Wilder, 1974). Men with these pains get more accident diagnoses, reflecting their higher injury rates.

Women who come for medication ( $\mathrm{SC1}$ ) or medical exams (GC1) are more likely to be diagnosed as sick than comparable men. This is partly because more of women's visits are actually for specific problems like obesity, genitourinary problems (previously diagnosed), and eye discomforts.

\section{Physician Sex Bias}

Only a few results suggest physician sex bias:

None of the other explanations fits these results for digestive, reproductive, nervous, general, and mental symptoms: First, physicians are more likely to say that women with these complaints are not sick, compared to men. Mental and digestive complaints also result in more "ill-defined" diagnoses. Do some physicians discount these symptoms entirely ('You are not sick") or partially ("You say you are sick, but I cannot find the medical problem, so I'll consider it ill-defined")? Second, headaches are attributed to mental and nervous conditions for women more than for men. Do physicians assume that headaches are psychogenic for women more often? ${ }^{13}$

Several commonly claimed biases do not appear. There is no overall sex difference in "symptomatic but not sick" status (hypochondria). Thus, physicians do not tend to say women are sick without good clinical evidence. Also, women do not appear to be overdiagnosed. They receive a similar number of diagnoses per complaint as men do.

\section{CONCLUSIONS}

Overall, how do the explanations for sex differences in complaints and diagnoses fare?

NAMCS data strongly support popular beliefs that men are more unaware of their health problems, that they delay seeking diagnosis and care for perceived symptoms, and that they are reticent to admit some symptoms and conditions when they do visit a physician.

There is not much evidence that men and women differ in their symptom perception, interpretation, and description. A few results do suggest that women have lower thresholds for symptom perception, know 
more about signs of serious diseases, and elaborate their problems more to physicians. Little evidence on these topics may reflect limitations of the NAMCS data more than lack of sex differences in the real world. Data which look directly at the dynamics of patient-physician encounters can answer these questions better. Moreover, NAMCS records are filled out by physicians, and patient complaints may not be recorded in the fullness or exact words that patients used.

The NAMCS data provide intriguing evidence that women have a more diffuse view of illness. The boundaries between physical and mental symptoms are probably less distinct for women than for men. And women do not pinpoint their physical symptoms in particular body sites as well as men; instead, the symptoms seem to radiate throughout the body.

Both sexes sometimes confuse reproductive, digestive, and urinary symptoms because the organs overlap physically.

Some results reflect differences in prevalence of health conditions for women and men. A particular symptom can reflect different conditions and thus lead to different diagnoses.

If physicians ultimately give different diagnoses to women and men for the same complaint, this may be entirely appropriate, showing awareness of the morbidity and social-psychological differences between the sexes. But sometimes the different outcome reflects sex bias-presumption of motives, needs, and health problems for women and men that influence diagnosis and treatment. A few NAMCS results look suspiciously like sex bias. They suggest that physicians sometimes view women's symptoms as psychogenic or discount their symptoms as signs of illness. But there is no evidence that physicians generally consider women to be hypochondriacal more than men. Overall, the evidence for sex bias in NAMCS data is scant. Stronger and more persuasive results are necessary to buttress popular claims that physician sex bias is a common feature of medical care.

In summary, there are eight plausible explanations for sex differences in complaints and diagnoses. Five involve social-psychological features of patients: symptom perception and interpretation, symptom description, diffuse view of illness, ignorance, and denial. Two involve physical features of patients: confusion of body systems, and different conditions for the same symptom. One involves social-psychological features of physicians: sex bias. The weight of evidence for explaining sex differences in NAMCS data is toward patient factors, especially their motives and attitudes.

Although this analysis focuses on sex differences, it is important to note how many similarities there are among women's and men's visits for ambulatory care. Most of the similarities were not discussed in the text. A few striking ones were: Women and men are equally likely to report a symptom of illness or injury, and equally likely to be diagnosed as "sick." The 
most common complaints and diagnoses tend to be the same. Overall, the numbers of symptoms reported and diagnoses given are comparable for both sexes. The concurrence of diagnosis with complaint is similar for women and men (with two noted exceptions). Both sexes receive the same number of diagnoses per complaint; neither is "overdiagnosed."

Thus, the sex differences found in NAMCS data appear in a broadly homogeneous context. This does not vitiate their importance. Taken together, they suggest some pervasive differences between women and men in motives for medical care, perspectives of illness, and possibly symptom perception and description.

NAMCS was not designed for extensive sociomedical analysis. But with appropriate caution, the data are a fine source for studying group differentials in complaints, diagnoses, services, and dispositions. Some interpretations can be tested within the NAMCS data themselves; others must be tested in other data sets with more information about patient and physician attitudes and perspectives.

Further analysis is planned on the NAMCS data, particularly on sex differences in services and dispositions. Variables such as physician specialty, prior visit status, age, seriousness, and chronicity will be included as control variables. A forthcoming paper determines whether women and men receive similar treatments for common complaints (Verbrugge and Steiner, 1980). This extends the recent work of Armitage et al. (1979), who found evidence that men receive more services and more appropriate ones for several common complaints. In addition, a working paper is available on male-female differences in mental health visits (symptoms of mental distress, diagnoses of mental disorder, services and dispositions for mental problems) (Verbrugge, 1979).

\section{REFERENCES}

Apple, D. (1960). How laymen define illness. J. Health Human Behav. 1: 219-225.

Armitage, K. J., Schneiderman, L. J., and Bass, R. A. (1979). Response of physicians to medical complaints in men and women. J. Am. Med. Assoc. 241(20): 2186-2187.

Drury, T. F. (1977). Current Estimates from the Health Interview Survey, United States, 1975. Vital and Health Statistics, Series 10, No. 115, National Center for Health Statistics, Hyattsville, Md.

Ehrenreich, B. (1974). Gender and objectivity in medicine. Int. J. Health Serv. 4(4): 617-623.

Epstein, F. J., Francis, T., Jr., Hayner, N. S. Johnson, B. C., Kjelsberg, M. O., Napier, J. A., Ostrander, L. D., Jr., Payne, M. U., and Dodger, H. J. (1965). Prevalence of chronic diseases and distribution of selected physiologic variables in a total community, Tecumseh, Michigan. Am. J. Epidemiol. 81(3): 307-322.

Feldman, J. (1966). The Dissemination of Health Information, Aldine, Chicago.

Fidell, L. S. (1980). Sex role stereotypes and the American physician. Psychol. Women Quar. 4(3): 313-330.

Freidson, E. (1973). Profession of Medicine, Dodd, Mead, New York. 
Hadden, W. F. (1978). Unpublished tabulations from the Health and Nutrition Examination Survey 1971-1974. Medical Statistics Branch, Division of Health Examination Statistics, National Center for Health Statistics, Hyattsville, Md.

Higgins, M. W., Keller, J. B., and Metzner, H. L. (1977). Smoking, socioeconomic status, and chronic respiratory disease. Am. Rev. Resp. Dis. 116: 403-410.

Howell, M. C. (1974). What medical schools teach about women. N. Engl. J. Med. 291: 304-307.

Koch, H, and McLemore, T. (1978). The National Ambulatory Medical Care Survey: 1975 Summary. Vital and Health Statistics, Series 13, No. 33, National Center for Health Statistics, Hyattsville, Md.

Lennane, K. J., and Lennane, R. J. (1973). Alleged psychogenic disorders in women-A possible manifestation of sexual prejudice. N. Engl. J. Med. 288(6): 288-292.

Levine, S., and Kozloff, M. A. (1978). The sick role: Assessment and overview. In Turner, R. H. (ed.), Annual Review of Sociology, 1978, Annual Reviews Inc., Palo Alto, Calif.

Maccoby, E. E., and Jacklin, C. N. (1974). The Psychology of Sex Differences, Stanford University Press, Stanford, Calif.

McCraine, E. W., Horowitz, A. J., and Martin, R. M. (1978). Alleged sex-role stereotyping in the assessment of women's physical complaints: A study of general practitioners. Soc. Sci. Med. 12: 111-116.

Meads, S., and McLemore, T. (1974). The National Ambulatory Medical Care Survey: Symptom Classification. Vital and Health Statistics, Series 2, No. 63, National Center for Health Statistics, Rockville, Md.

Mechanic, D. (1972). Social psychologic factors affecting the presentation of bodily complaints. N. Engl. J. Med. 286(21): 1132-1139.

National Center for Health Statistics (1967). Eighth Revision International Classification of Diseases, Adapted for Use in the United States, PHS Publ. No. 1693, Government Printing Office, Washington, D.C.

Notermans, S. L. H., and Tophoff, M. M. W. (1967). Sex differences in pain tolerance and pain apperception. Psychiat. Neurol. Neurochirurg. 70: 23-29.

Scheff, T. J. (1966). Being Mentally Ill, Aldine, Chicago.

Schneider, D., Appleton, L., and McLemore, R. (1979). A Reason for Visit Classification for Ambulatory Care. Vital and Health Statistics, Series 2 , No. 78, National Center for Health Statistics, Hyattsville, Md.

Suchman, E. A. (1965a). Stages of illness and medical care. J. Health Human Behav. 6(3): 114-128.

Suchman, E. A. (1965b). Social patterns of illness and medical care. J. Health Human Behav. 6(1): 2-16.

Tenney, J. B., White, K. L., and Williamson, J. S. (1974). National Ambulatory Medical Care Survey Background and Methodology. Vital and Health Statistics, Series 2, No. 61, National Center for Health Statistics, Rockville, Md.

Verbrugge, L. M. (1979). Sex differences in mental health-Complaints, diagnoses, services, and dispositions, Unpublished paper (available from author).

Verbrugge, L. M. (1981). Sex differentials in health and mortality. In Stromberg, A. H. (ed.), Women, Health, and Medicine, Mayfield, Palo Alto, Calif.

Verbrugge, L. M., and Steiner, R. P. (1980). Differences in physician treatment of men and women-Sex bias or appropriate care? Paper presented at the 1980 meetings of the American Public Health Association, Detroit, October.

Wallen, J., Waitzkin, H., and Stoeckle, J. D. (1979). Physician stereotypes about female health and illness: A study of patients' sex and the information process during medical interviews. Women and Health 4(2): 135-146.

Wilder, C. S. (1973). Prevalence of Selected Chronic Respiratory Conditions, United States, 1970. Vital and Health Statistics, Series 10, No. 84, National Center for Health Statistics, Rockville, Md.

Wilder, C. S. (1975). Prevalence of Chronic Circulatory Conditions, United States, 1972. Vital and Health Statistics, Series 10, No. 94, National Center for Health Statistics, Rockville, Md. 
Wilder, M. H. (1974). Prevalence of Chronic Skin and Musculoskeletal Conditions, United States, 1969. Vital and Health Statistics, Series 10, No. 92, National Center for Health Statistics, Rockville, Md.

Woodrow, R. M., Friedman, G. D., Siegelaub, A. B., and Collen, M. F. (1972). Pain tolerance: Differences according to age, sex, and race. Psychosom. Med. 34: 548-556. 